NP28 (continued)

presented challenges for Extension personnel who deliver the programs. Strategies for randomization were revised to inform Extension personnel of which program their church will receive earlier, prior to baseline data collection, to allow more time for preparation and scheduling. Research and church personnel are blinded to condition prior to baseline data collection.

Funding: 2018-68001-27549.

\section{NP29 The Family Check-Up 4 Health: A Health Maintenance Approach to Improve Nutrition and Prevent Early Childhood Obesity}

Cady Berkel,PhD, cady.berkel@asu.edu, Arizona State University, 148 W Vera Ln, Tempe, AZ 85284; Justin D. Smith, PhD, Northwestern University; Emily Fu, MPH, Northwestern University; Meg Bruening, PhD, MPH, RN, Arizona State University; Thomas Dishion, $P h D$, Arizona State University

Objective: We will test the effectiveness of the Family Check-Up 4 Health (FCU4Health) intervention in a type I hybrid trial to simultaneously evaluate clinical effectiveness and the implementation process.

Description: FCU4Health is an adaptation of the Family Check-Up (FCU), an evidence-based family-centered intervention. The program targets parenting behaviors to improve child nutrition and other family health routines. It includes a brief initial interview, an assessment of the child and family, and a feedback and motivation session for all families, followed by family support intervention(s) that are individually-tailored in content and dosage based on needs identified in the assessment. Family support sessions are derived from Everyday Parenting, a 12-module skills-based curriculum focusing on relationship quality, positive behavior support, and monitoring and limit setting. FCU4Health has been tested in a randomized hybrid II trial conducted in partnership with three primary care clinics to address obesity for patients 6-12 years old with elevated BMI ( $\geq 85$ th percentile). The current study focuses on obesity prevention by improving childhood nutrition among 2-5-year-olds. Specifically, we will deliver the FCU4Health three times (annually) to diverse, lowincome families. Each year, families will complete a comprehensive assessment, parent training modules that address their specific parenting needs, and referrals to community resources to address contextual needs (e.g., food insecurity).

Evaluation: We will conduct a type I hybrid effectiveness and implementation trial of FCU4Health in a population of predominantly low-income and Latino families. Families will be recruited from an integrated behavioral health and primary care clinic, preschool, and a family resource center and randomized to the intervention $(n=130)$ or usual care $(n=70)$. Multiple implementation outcomes, including acceptability, feasibility, fidelity, and reach will be assessed and the economic impact of FCU4Health will be evaluated.
Conclusion and Implications: The FCU4Health is unique in its approach to improving pediatric nutrition through a focus on parenting. It is also one of the first parenting interventions to be delivered in primary care.

Funding: 2018-68001-27550.

\section{NP30 Children's Healthy Living Center of Excellence (CHL Center) Year 1}

Rachel Novotny, PhD, LD, RDN, novotny@hawaii.edu, University of Hawaii at Manoa, 1955 East West Rd, Honolulu, HI 96822; Marie Fialkowski, PhD, LD, RDN, University of Hawaii at Manoa; Jean Butel, PhD, University of Hawaii at Manoa; Travis Fleming, RDN, American Samoa Community College; Leslie Shallcross, RDN, University of Alaska at Fairbanks; Jonathan Deenik, PhD, University of Hawaii at Manoa; Patricia Coleman, BA, Northern Marianas College; Rachael LeonGuerrero, PhD, RDN, University of Guam

Objective: To serve as a Center of Excellence to further build capacity and new knowledge for collective impact on child health in the US Affiliated Pacific Region.

Description: CHL Center continues to strengthen capacity with the Child Health in the Pacific (CHAP) Summer Fellowship Program and the CHL Dietetics Scholarship Program (CHLD), the CHL Summer Institute. Ten fellows from CNMI, Guam and Hawai'i completed the CHAP Summer Fellowship program and the CHL Summer Institute had a total of 123 students completing online courses (nine offered) related to childhood obesity. To examine the CHL intervention's long-term impact, retraining and standardization workshops are being conducted; and data collection has begun. Eighteen community reports provide a comprehensive compilation of data already collected, and CHL data are available for continued analysis to fill the void in basic nutrition and health information of children in the region. A CHL network multistate project (W1194) has expanded CHL's reach, with members from Arizona and West Virginia.

Evaluation: The published $\mathrm{CHL}$ intervention effect showed significant improvement in intervention communities compared with control in overweight and obesity prevalence (effect size [d] $=-3.95 \% ; 95 \% \mathrm{CI},-7.47 \%$ to $-0.43 \%)$, waist circumference $(\mathrm{d}=-0.71 \mathrm{~cm}$; $95 \% \mathrm{CI}$, -1.37 to $-0.05 \mathrm{~cm}$ ), and acanthosis nigricans prevalence ( $d=-2.28 \%$; 95\% CI, $-2.77 \%$ to $-1.57 \%$ ). Greater differences existed among intervention communities in acanthosis nigricans prevalence in the group aged 2 to 5 years $(-3.99 \%)$ vs the group aged 6 to 8 years $(-3.40 \%)$, and the interaction was significant $(\mathrm{d}=0.59 \%, P<.001)$. There was a smaller difference in the group aged 2 to 5 years $(-0.10 \%)$ vs the group aged 6 to 8 years $(-1.07 \%)$ in screen time $(\mathrm{d}=-0.97$ hour per day, $P=.01)$. Further analysis of intervention levels, activities and child behaviors that influenced the intervention effect continues to be investigated.

Conclusion and Implications: Year one built on efforts established by the CHL program to build the CHL Center of Excellence.

Funding: 2018-68001-27551. 